\title{
Innovations in total knee replacement: new trends in operative treatment and changes in peri-operative management
}

\author{
Stefano Zanasi
}

Received: 15 March 2011 / Accepted: 6 June 2011 /Published online: 13 July 2011

(C) The Author(s) 2011. This article is published with open access at Springerlink.com

\begin{abstract}
The human knee joint can sustain damage due to injury, or more usually osteoarthritis, to one, two or all three of the knee compartments: the medial femorotibial, the lateral femorotibial and the patellofemoral compartments. When pain associated with this damage is unmanageable using nonsurgical techniques, knee replacement surgery might be the most appropriate course of action. This procedure aims to restore a pain-free, fully functional and durable knee joint. Total knee replacement is a wellestablished treatment modality, and more recently, partial knee replacement-more commonly known as bi- or unicompartmental knee replacement-has seen resurgence in interest and popularity. Combined with the use of minimally invasive surgery (MIS) techniques, genderspecific prosthetics and computer-assisted navigation systems, orthopaedic surgeons are now able to offer patients knee replacement procedures that are associated with (1) minimal risks during and after surgery by avoiding fat embolism, reducing blood loss and minimising soft tissue disruption; (2) smaller incisions; (3) faster and less painful rehabilitation; (4) reduced hospital stay and faster return to normal activities of daily living; (5) an improved range of motion; (6) less requirement for analgesics; and (7) a durable, well-aligned, highly functional knee. With the ongoing advancements in surgical technique, medical
\end{abstract}

This review is based on an oral presentation given by S. Zanasi at the satellite symposium entitled From Proof to Practice: Innovations in Total Hip and Total Knee Replacement, which was held during the 11th EFORT Congress, Madrid, Spain (2 June 2010).

\section{S. Zanasi $(\bowtie)$}

Third Division-MIS Hip and Knee Joint Arthroplasty Operative Centre, Orthopaedics Department, Gruppo San Donato,

Villa Erbosa Hospital,

Bologna, Italy

e-mail: zanasis.orth@alice.it technology and prosthesis design, knee replacement surgery is constantly evolving. This review provides a personal account of the recent innovations that have been made, with a particular emphasis on the potential use of MIS techniques combined with computer-assisted navigation systems to treat younger, more physically active patients with resurfacing partial/total implant knee arthroplasty.

Keywords Minimally invasive surgery. Oral anticoagulants · Orthopaedics · Rehabilitation .

Total knee replacement . Venous thromboembolism

\section{Introduction}

The knee is the largest joint in the body, consisting of two articulations: one between the femur and tibia, and one between the femur and patella. The majority of the body's weight is supported by the knee joint and as a consequence it is often affected by acute injury or iterative microtraumas, with the development of osteoarthritis in later life. The anatomy of the knee is divided into three compartments: the inner medial femorotibial compartment; the outer lateral femorotibial compartment; and the compartment that consists of the patella (kneecap) and the femur, the patellofemoral (PF) compartment. Damage, due to injury or more usually osteoarthritis, can occur to one, two or all three of these knee compartments.

The main reason for considering knee replacement surgery is to relieve the pain caused by osteoarthritis once all other non-surgical interventions have been shown to be unsuccessful. Surgery aims to reconstruct/resurface a painfree joint that maintains the best proprioceptivity and performance. When considering knee replacement surgery, orthopaedic surgeons have the option to undertake a total 
knee replacement (TKR) or a partial knee replacement [also known as a unicompartmental knee replacement/resurfacing (UKR) or bi-unicompartmental knee resurfacing] (Fig. 1). For example, if a case presents with osteoarthritis in just one compartment, usually the medial femorotibial compartment, the orthopaedic surgeon could choose to replace the entire knee joint or to resurface just the affected compartment (i.e. tailored resurfacing implant arthroplasty). Patients with severe damage that warrants TKR rather than UKR often present with damage in two or more compartments. TKR has a finite survival rate, dependent mainly upon the implant alignment and post-operative activity level of the recipient $[1,2]$, so is often used in elderly patients with limited activities or in younger, low-demand patients who have limited function because of systemic disease in multiple joints.

After a period of diminishing popularity [3, 4], interest in UKR has increased in recent years, with the recognition that overcorrection of the mechanical axis must be avoided and the publication of studies showing high rates of longterm (10-15 years) joint survival [5-7]. Although generally considered a more difficult procedure than TKR, UKR is thought to allow preservation of the uninvolved soft tissue and bone, reduced operating time, better post-operative range of motion, less pain, better stair-climbing ability, improved gait due to proprioceptivity maintenance and increased patient satisfaction than TKR [8,9]. With appropriate patient selection and careful surgical technique, UKR can provide the following advantages over TKR: (1)

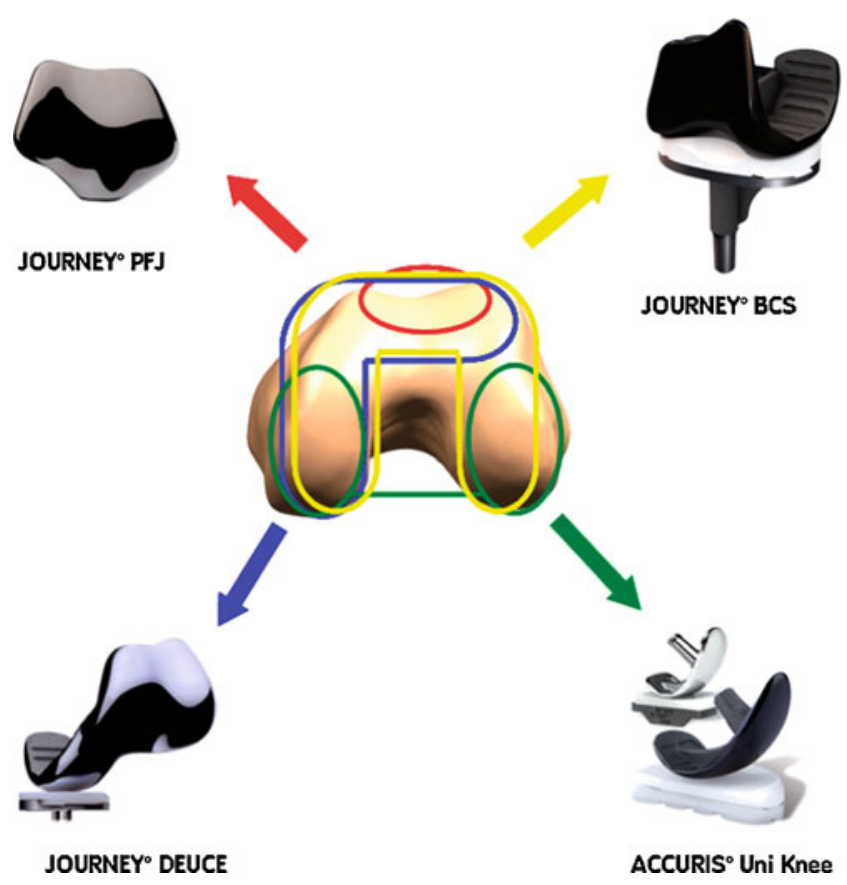

Fig. 1 In uni-/bi-/three-compartmental osteoarthritis involvement, a new approach is resurfacing combination arthroplasty, i.e. with oxinium implant arthroplasty smaller incision if a minimally invasive surgery (MIS) technique is used; (2) easier post-operative rehabilitation; (3) shorter hospital stay; (4) less blood loss; (5) lower risk of infection; (6) less joint stiffness; (7) lower risk of venous thromboembolism (VTE) due to non-invasion of the medullary space; and (8) easier revision surgery, if required, at a later date.

During the $30-50$ years that UKR and TKR have been practised, there have been considerable improvements in surgical techniques and in the type and quality of available prosthetic joints. The techniques that have evolved maximise implant performance and longevity, whilst providing a rapid return to normal standing and mobilisation. This paper will review a number of cases to illustrate the range of surgical techniques, which are suited to particular patient populations, based on the level of joint involvement and the patient's clinical characteristics.

\section{MIS for knee replacement}

MIS for both TKR and UKR/bi-UKR generally involves the following: (1) a shorter incision length $(10-13 \mathrm{~cm}$ compared with $15-23 \mathrm{~cm}$ for TKR); (2) retraction of the patella without eversion or rotating out; (3) sparing of the quadriceps tendon by not cutting the quadriceps femoris muscle from the patella and not violating the suprapatellar pouch; (4) the use of smaller, specialised instruments that provide accuracy through a smaller anteromedial surgical window; and (5) the creation of a mobile surgical window that minimises soft tissue tension and positions the exposure over the anatomical region of interest [10-13]. In particular, the mini-midvastus MIS approach is considered the gold standard technique and can be used in all types of patients, giving the surgeon a very good view of the knee and allowing a range of surgical procedures to be undertaken [14].

There are few randomised studies comparing MIS and traditional surgical techniques, but the consensus is that MIS provides several advantages, such as the requirement for less muscle dissection, less blood loss, less pain, a shorter hospital stay, faster rehabilitation and a more rapid improvement in the range of motion [12, 13, 15-17]. Recuperation following traditional surgery for knee replacement is often arduous and painful, and it is usually not possible to regain a full range of motion despite aggressive rehabilitation regimens. In our experience, by adopting a MIS approach for both TKR and UKR, we can reduce the incidence of complications such as fat embolism, preserve the bone stock and to cause minimal damage to the soft tissues, whilst maintaining/restoring optimal capsuleligamentous balancing of the knee joint. This then translates into a number of advantages for the patient, including fast 
and maximal recovery of the range of motion; painless, shorter recovery time and cosmetic benefits due to the limited skin incision. Care taken during the MIS procedure should ensure that if a revision is required at a later date, it will not be adversely affected by this earlier surgery.

\section{Isolated medial or lateral unicompartmental involvement requiring medial or lateral unicompartmental knee arthroplasty}

The ideal indications for UKR proposed by Kozinn and Scott [18], revised more recently by several authors, and in association with new designs and materials, have resulted in higher success rates. Careful patient selection is critical for unicompartmental knee arthroplasty if reliable results are to be achieved. The arthritis should be predominantly confined to a single compartment. No significant degenerative changes in the other (medial, lateral or PF) compartments should be present, and both cruciate ligaments should be intact. Absence of the anterior cruciate ligament (ACL) is a contraindication; the ACL makes the combined rolling and sliding at the meniscal femoral and meniscal tibial interfaces possible, which may yield near-normal joint kinematics and mechanics. The operation is also indicated in patients with osteonecrosis of the femoral condyle. Not all of the unicompartmental replacements are suitable for the lateral side because the ligaments of the lateral compartment are more elastic than those of the medial side. Malalignment of the limb should be passively correctable to neutral and not beyond. This is usually possible in patients with a varus deformity $<15^{\circ}$ or a valgus deformity $<20^{\circ}$. The deformity of the knee should be only mild; therefore, a flexion contracture should be $<15^{\circ}$. Unicompartmental knee arthroplasty with excision of osteophytes in the notch cannot correct moderately severe flexion contractures. Ideally, the knee can be flexed to $110^{\circ}$. This is important for the preparation of the femoral condyle during the operation. Recently survival rates of $>90 \%$ at 10 years' follow-up have been shown even in patients $<60$ years of age by Swienckowski et al. [19]. In comparison with TKR, UKR allows use of smaller implants, shorter operative time, and preservation of both the cruciate ligaments and minimal bone resection [20,21]. Maintenance of the ACL and its mechanoreceptors may produce a better functional result in UKR [22-24]. Knee kinematics during flexion following UKR have been shown to more closely resemble the intact knee; however, biomechanical studies of TKR have yielded results far from that of a normal knee [22, 25]. Weale et al. [26] documented superior functional recovery with improved performance in descending stairs and better patient satisfaction with UKR compared with TKR. In a cadaveric study, Patil et al. [27] demonstrated normal joint biomechanics after
UKR implantation in a knee. A number of prosthetic designs are now available, including both mobile and fixed tibial bearing surfaces: no statistically significant clinical advantage could be demonstrated between a fixed or mobile bearing tibial component in UKR at a mean follow-up of 5.7 years [28], or 6.8 and 7.7 years [29]. We have had good success in treating patients with either medial or lateral unicompartmental disease using the MIS Unicompartmental Fixed Knee System (ZUK, Zimmer ${ }^{\circledR}$ Inc., Warsaw, IN, USA; JOURNEY OXINIUM UNI, Smith \& Nephew, Memphis, TN, USA). Typically, patients recover functionality of the operated knee within 40 days.

\section{Unicompartmental isolated PF involvement requiring unicompartmental PF knee arthroplasty}

Approximately $5-10 \%$ of patients with osteoarthritis of the knee will have isolated unicompartmental PF involvement and therefore would be good candidates for PF replacement (PFR) [30, 31]. During these procedures, plastic buttons can be used to resurface the patella before it is put back into place. A metallic shield is placed in the trochlear groove of the femur. Because of variable outcomes, at present, PF knee arthroplasty remains a controversial treatment for advanced osteoarthritis. Many surgeons perform total knee replacement for isolated advanced PF osteoarthritis, rather than PF arthroplasty, as means of achieving more consistent outcomes. Some of the current issues surrounding isolated PF arthroplasty are the fact that extensive exposure is necessary, a lack of long-term outcome studies and the variable success rate of this procedure [32]. The clinical results and 10-year survival rate have been good with some modular unicompartmental knee designs of PF knee arthroplasty, although radiographic signs of progression of osteoarthritis in the other compartments have been found to continue at a slow rate and progressive PF arthritis is the primary mode of failure [6]. Various authors have reported excellent results following total knee arthroplasty for the treatment of isolated PF arthritis in elderly patients, but young patients with advanced isolated $\mathrm{PF}$ arthritis who are not candidates for osteotomy, patellectomy, isolated patella resurfacing or total knee arthroplasty still represent a challenge for the orthopaedic surgeon. PF arthroplasty is an attractive alternative to TKR with potential advantages in this group. The ideal indication is a truly isolated PF arthritis with a varus deformity no greater than $5-6^{\circ}$ and a valgus deformity of $7-8^{\circ}$, according to Witvoet [31]. With the correct indications and surgical technique, good results can be obtained in more than $80 \%$ of patients. In our experience, this procedure gives the patient the possibility of regaining complete weight-bearing at 10-12 days post- 
operation and a very good range of motion within 30 days without pain

\section{Bi-unicompartmental involvement requiring bi-unicompartmental knee arthroplasty}

When osteoarthritis affects either the medial or lateral compartments of the knee, we now have the option to undertake a bi-unicompartmental knee replacement instead of a TKR. This procedure uses two independent femoral, and two independent tibial, prosthetic components (Fig. 2). A matched paired study between bi-unicompartmental versus TKR for the treatment of isolated bicompartmental tibio-femoral knee arthritis with an asymptomatic PF joint showed that at a minimum follow-up of 48 months there were no statistically significant differences in function between the two groups, although there was indication that bi-unicompartmental knee replacement may maintain a higher level of function [33]. In our experience, this procedure provides the same advantages as UKR over TKR as shown by (1) preservation of the intercondylar eminence with both of the cruciate ligaments, (2) maintenance of the rotational axis, (3) preservation of bone stock, (4) normal patella level and tracking, (5) restoration of normal kinematic and gait, (6) maintenance of normal leg morphology and (7) maintenance of normal proprioception. This technique is difficult because it requires reproduction of the anatomy of each individual patient's knee; however, it is possible to obtain very good results, with patients being able to achieve a knee flexion of $135^{\circ}$ by day 10 post-operation.
Most total knee arthroplasty designs have kinematics which differ from the normal knee, while unicondylar knee arthroplasties have shown nearly normal knee kinematics. Cruciate retention and $\mathrm{PF}$ intact compartments in biunicondylar knees are more likely to provide normal control of knee motion [34]. It is unclear whether retention of both cruciates in a bicompartmental arthroplasty is sufficient to provide similar motions to that of the normal knee. Intrinsic knee stability is directly linked to functional performance, both in people who practice sport and in those who have had arthroplasty; bicruciate retaining knee arthroplasty might provide more normal knee motions and functional benefits compared with total knee arthroplasty which retains only one or none of the cruciate ligaments. Retaining both cruciate ligaments in resurfacing knee arthroplasty appears to maintain the essential features of the normal knee motion: femoral rollback and tibial internal rotation with flexion: bicruciate retaining knee arthroplasty, even if it is not commonly performed, appears to provide a high level of function and knee kinematics in patients retaining essential features of the normal knee [24, 34]. Fuchs et al. [24] reported that implants preserving both the cruciate ligaments can achieve functional results at least similar to TKR without any arthritis progression.

This type of surgery is indicated for patients with bilateral femorotibial degeneration but with an asymptomatic patella, with cruciate ligament integrity, flexum deformity $<5^{\circ}$, varus-valgus deformity $<15^{\circ}$ and range of motion $>80^{\circ}$ [34]. Radiographical evaluation is based on AP, lateral and sky view projections that show femorotibial degeneration higher than grade II on the Ahlback scale, and PF involvement lower than grade II. Magnetic resonance

Fig. 2 Photographs showing the bi-unicompartmental knee replacement components used to resurface the medial and lateral compartments of the knee; anterior and lateral X-rays showing the osteoarthritic knee joint prior to surgery (a) and the resurfaced medial and lateral compartments of the right knee joint after surgery (b); the postoperative X-ray film (c), and degree of knee flexion $\left(135^{\circ}\right)$ achieved by the same patient at 9 days post-operation (d)
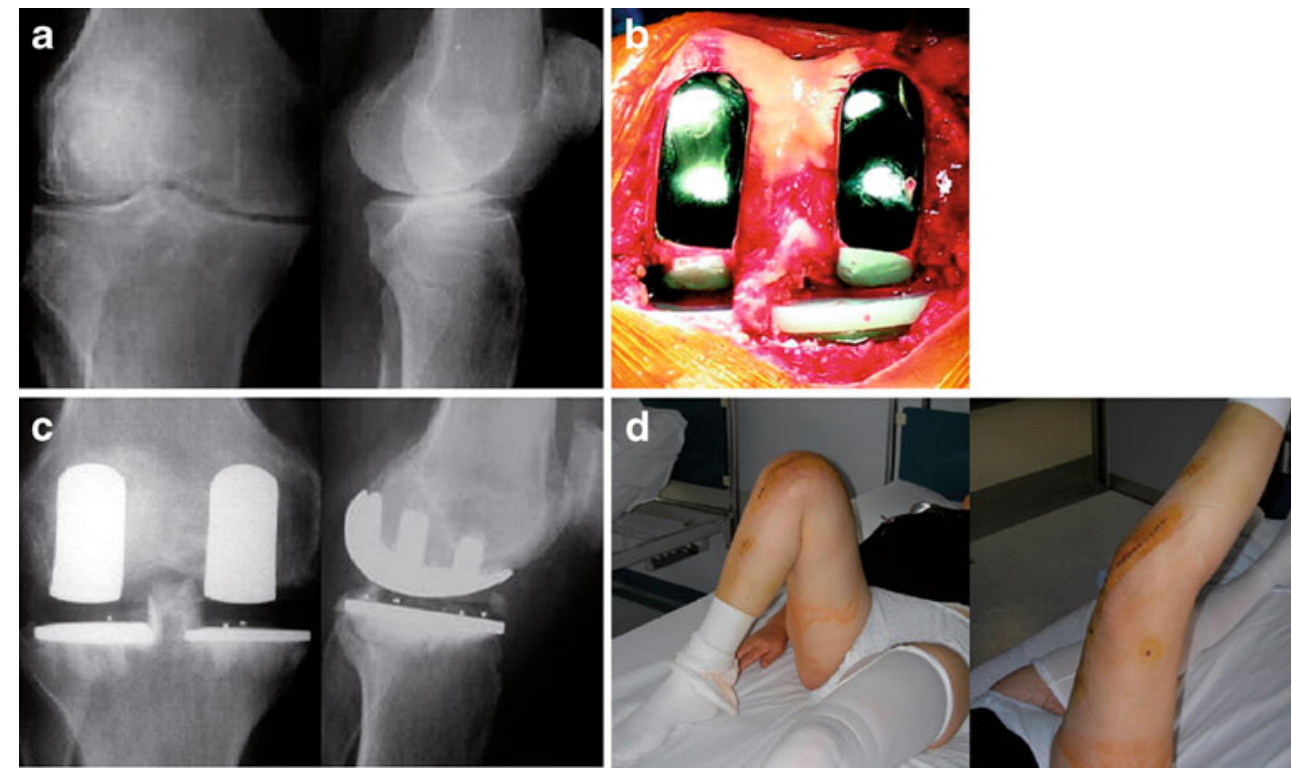
imaging can highlight both ACL instability or deficiency, and PF degeneration. The knee must be stable clinically, and only a minimal laxity, due to cartilaginous degeneration, is tolerated. Main clinical signs are pain while walking and climbing stairs, and effusion. Although, age and weight are not limitations, this procedure is especially suitable for active patients $<65$ years of age and with a body mass index (BMI) <32. Bi-unicompartmental is, in fact, suitable for young patients with high functional expectations. As previously mentioned, the main limitations of this implantation selection are ACL and PF integrity. In the first case, when femorotibial bicompartmental degeneration defines a correct indication for a bi-unicompartmental implant, but the absence of ACL is a clear limitation, only then can we consider an ACL reconstruction.

One of the most common causes of failure using this procedure is surgical error due to difficult surgical technique, malposition of the components, intercondylar eminence fracture and wrong ligament balance, and cementation mistakes. In our experience, possible causes of failure are femoropatellar degeneration and acquired instability. Underestimated preoperative pain or post-operative degeneration of the femoropatellar joint can lead to revision surgery for continuous anterior knee pain even in well-performed biUKR. In this case, according to Romagnoli [35], the easiest solution is to implant femoropatellar prosthesis, if all components are stable and with no wear. The alternative solution is to change femoral components, substituting them with a total femur prosthesis. A traumatic rupture of the ACL is another cause of failure especially in young patients who return to sporting activity after surgery, and this cause, together with post-operative instability in the wrongly balanced knee, can be the cause of revision of TKR. Finally, infection loosening has the same incidence as in other prosthetic procedures.

The benefits of this approach when compared to TKR include greater tissue sparing, reduced surgical morbidity and easier revision surgery. Current patient expectations following knee replacement surgery include a knee resembling normal appearance, and one which allows an unrestricted, active life. Because of the superior biomechanical resemblance of the bi-UKR to a normal knee, it may better match these expectations. Despite these potential advantages, no series of bi-unicompartmental knee replacement has been reported in the literature.

\section{Bicompartmental involvement requiring $\mathbf{P F}$ and unicompartmental knee arthroplasty}

Knee arthritis often involves only one of the two femorotibial compartments, along with symptomatic PF joint degeneration. Treatment of bicompartmental arthritis involves a lateral or medial UKR to treat arthritis and correct the axial deformity, as well as the use of PF prosthesis [36]. Small-scale studies have reported this to be a successful approach to prevent or postpone TKA [37], although as with most of the newer techniques there is a lack of long-term, evidence-based studies. Combined use widens the indications and reduces the limitations of UKR and isolated PF prosthesis. This procedure is suitable in cases of borderline UKR indications with femorotibial compartmental arthritis and symptomatic patella, and in cases of borderline indications for PF prosthesis due to isolated $\mathrm{PF}$ arthritis with $3^{\circ}$ mechanical axis deviation and initial femorotibial unicompartmental involvement. The isolated PF prosthesis is an uncommon procedure with few references in the literature and even rarer is its combined use with UKR. The advantages of a bicompartmental implant, UKR plus PF, are cruciate preservation, respect for rotational axis, bone stock preservation, patellar height and tracking, normal joint kinematic reproduction and morphotype respect. Selection criteria are the same as for UKR, in which the PF joint is degenerated. The objective in the surgical procedure is to respect the femoral surfaces' rotational axis and trochlear depth, avoiding excessive tension on the patella. Implants must highlight perfect PF tracking without patellar clunk and tilting in the area of the component transition.

\section{Bicompartmental involvement requiring bicompartmental knee arthroplasty}

In certain patients, the medial and PF compartments are affected by osteoarthritis, but the ACL and lateral compartment remain healthy. These patients are candidates for bicompartmental knee replacement. Bicompartmental arthroplasty preserves all of the ligaments of the knee while replacing two compartments [38]. The procedure is more complicated than unicompartmental knee arthroplasty, less invasive than total knee arthroplasty and may have a place in replacement surgery, although long-term evidence is lacking. Bicompartmental arthroplasty is reported to offer decreased pain, stability through normal ligament structure, and the retention of normal bone for patients with medial and patellofemoral osteoarthritis [39]. We have had success using a solid prosthetic component to cover the two compartments (Fig. 3), which is not a TKR. Compared with unicondylar knee arthroplasty and TKR, the JOURNEY DEUCE Bi-Compartmental Knee System (Smith \& Nephew, USA) could be described as the ideal knee implant for patients with mid- to late-stage arthritis involving the medial and PF compartments. JOURNEY DEUCE is a durable device available in low-wear oxidised zirconium for the high-demand patient. The device uses a monolithic design 
Fig. 3 Photographs showing the surgical procedure to insert the solid bicompartmental knee replacement component used to resurface the medial and PF compartments of the knee (a); lateral and anterior X-rays showing the resurfaced medial and PF compartments of the left knee (b); the degree of knee flexion $\left(140^{\circ}\right)$ achieved by the same patient at 12 days post-operation (c)
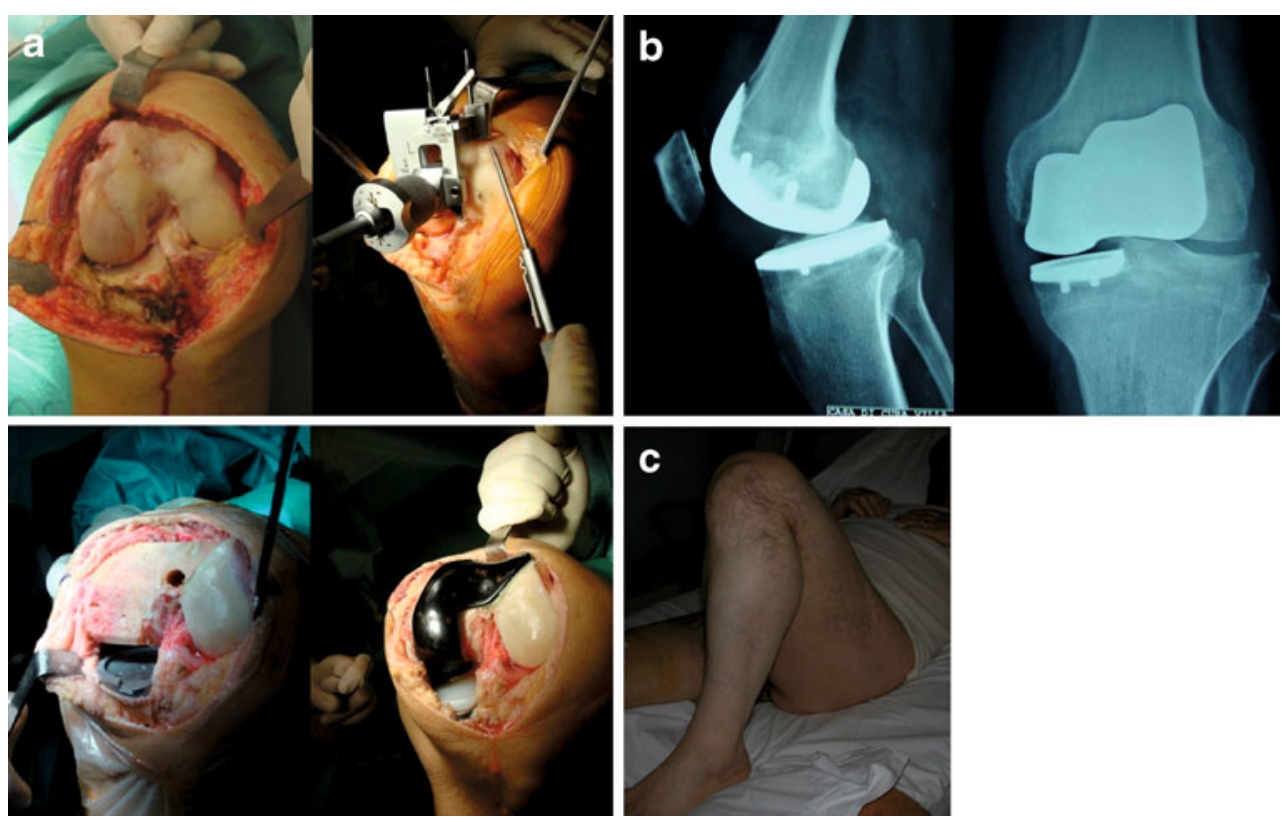

that replaces only the diseased medial and PF compartments, while sparing the lateral compartment and both cruciate ligaments. Retention of the cruciate ligaments maintains normal kinematics and proprioception. Because bone and ligaments are conserved, pain relief should be similar to unicondylar knee arthroplasty. Less pain and reduced tissue trauma help to speed up recovery and a return of function. Conservation of bone also provides reference landmarks in the event of future revision. JOURNEY DEUCE is suited for the active, high-demand patient and also benefits the less active patient, regardless of age. Contraindications for this bicompartmental arthroplasty include tricompartmental arthritis, rheumatoid arthritis, fixed flexion contracture, severe deformity and functional ACL laxity. In our experience, patients can achieve $140^{\circ}$ of knee flexion and are able to walk without the use of crutches by day 12 post-operation. In the literature, there is only a short-term ( 33 months) follow-up of 95 cases performed in a pilot study with this new implant $[39,40]$. The authors reported no revision with a high level of satisfaction following implant.

\section{Three compartmental involvement requiring total knee arthroplasty}

When all three compartments of the knee are affected by osteoarthritis, a traditional TKR is appropriate. With the current designs of TKR prostheses and surgical techniques, we would expect our patients to regain a range of motion of the knee from $0^{\circ}$ to $110^{\circ}[41,42]$, falling short of the normal range of motion of a healthy knee $\left(0-145^{\circ}\right)$. The restricted motion following traditional TKR can have a major impact on patients because they live in cultures where squatting and sitting on the floor are essential activities of their daily lives. In these patients, a deep flexion of the knee $\left(>120^{\circ}\right)$ may be required, but achieving this goal whilst maintaining stability of the knee joint is challenging. It must be borne in mind that the best predictor of the post-operative range of motion is the preoperative range of motion [43]. Patients will never achieve a greater range of motion of the knee than they had prior to surgery. Additionally, surgical technique, prosthesis design and post-operative rehabilitation can influence the range of motion following TKR. During the procedure, we try to spare the bone by removing no more than $4-5 \mathrm{~mm}$ from the tibial plate and no more than 3$4 \mathrm{~mm}$ from the femoral plate so that the total resection does not exceed $12 \mathrm{~mm}$. Using the Zimmer ${ }^{\circledR}$ NexGen ${ }^{\circledR}$ LPS-Flex tantalum ultra-high molecular weight polyethylene resurfacing TKR system (Zimmer ${ }^{\circledR}$ Inc.), we would expect our patients to regain the full range of motion of the knee within 40 days post-operation. However, surgical techniques and prosthetic designs are constantly being improved, enabling us to address new challenges that would not have been possible previously. For example, we have had success during the past 3 years with a concurrent bilateral TKR. Figure 4 shows the case of a young, fullfigured woman, who was weight-bearing and walking without aids within 3 days of the operation with concurrent bilateral total knee resurfacing arthroplasty. 

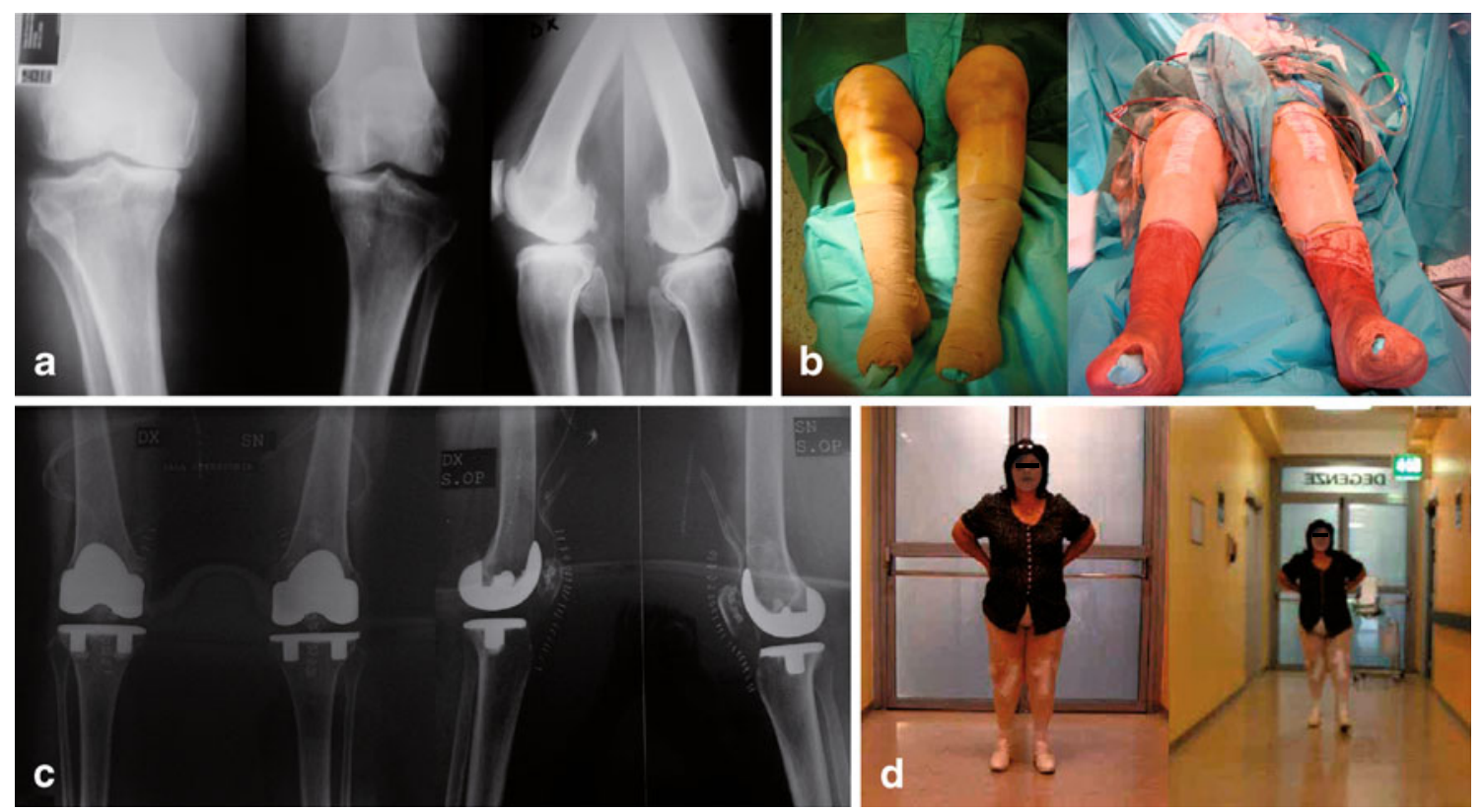

Fig. 4 a Preoperative bilateral X-ray films; $\mathbf{b}$ immediate preoperative and post-operative outcome; $\mathbf{c}$ post-operative X-ray films; d deambulation without any cane at fourth day post-operative

\section{Gender solutions}

It is important to take account of gender differences in the anatomy of the knee when undertaking knee replacement surgery. For example, females have a smaller medial lateral femoral width than males for the same anterior-posterior femoral height. Females have a less prominent anterior condyle and they have a relatively more internally rotated distal femur than males. Females also have a more trapezoidal distal femur compared with males, who have a more rectangular one. The Zimmer ${ }^{\circledR}$ Gender Solutions ${ }^{\mathrm{TM}}$ NexGen ${ }^{\circledR}$ High-Flex Knee implant (Zimmer ${ }^{\circledR}$ Inc.) takes account of such anatomical differences. For example, it has a thinner profile than traditional implants, which can benefit range of motion and reduce the likelihood of pain compared with more bulky implants. This implant provides more natural movement by taking account of the different angles between the hip and the knee in females compared with males. It also has a contoured shape that prevents the implant overhanging the bone and potentially pressing on, or damaging, the surrounding ligaments or tendons. One 1-year outcomes of the first 360 patients who received the Zimmer Gender Solutions Natural-Knee Flex System found a mean flexion of $131^{\circ}\left(10^{\circ}\right.$ better than mean flexion with the earlier Natural-Knee I and II systems) and a decrease in the lateral release rate to $2 \%$ [44]. It was concluded that there was no need to downsize during surgery to achieve good fit in femurs of female patients who typically have narrower medial-lateral/anterior-posterior aspect ratios.
However, at 5 years' follow-up of 245 patients with Innex $\left(\right.$ Zimmer ${ }^{\circledR}$ ) mobile bearing total knee arthroplasty systems, male knees exhibited better clinical function and men reported greater satisfaction than their female peers despite inferior radiographic findings and higher revision rates [45].

\section{Navigation to assist limb alignment during total knee replacement}

Correct positioning of the components of the knee implant, limb alignment and balancing of the soft tissues are crucial to the subsequent recovery of a fully functional and durable knee joint [46]. Knee implants that have been placed in a suboptimal position are more likely to loosen and then fail. Correct positioning ensures that the weight is transmitted from the centre of the femoral head down through the centre of the knee joint and then to the centre of the ankle; this is known as the mechanical axis. Deviations from a neutral mechanical axis can cause joint loosening and failure $[47,48]$. However, using traditional techniques, the final position of the implanted prosthesis cannot be assessed accurately until an X-ray is taken after surgery. Although there are mechanical alignment guides that can assist the surgeon intra-operatively to cut the bone at the required angle, once the bone is cut, the optimal positioning of the implant is dependent on the components [49, 50], and upon the skill and judgement of the surgeon. Incorrect implant placement is thought to occur in more than $10 \%$ of 
TKRs performed by experienced surgeons [51], so computer-assisted surgical (CAS) navigation systems have been developed to assist in the correct alignment of the prosthetic components [49, 50]. These CAS navigation systems provide intra-operative, real-time information on the location of the joint centres, the position of the prosthetic implants and the alignment of the limb [49]. They can be used to guide the placement of the cutting blocks and they can also track surgical tools (Fig. 5) [49]. Navigation systems also allow the intra-operative assessment of the range of motion of the knee and kinematics [46]. They enable the prosthetic knee to be positioned closest to the individual's normal anatomy. CAS navigation systems have been shown in clinical trials to improve the accuracy of bone resections and mechanical axis alignment of the limb in TKR, and recent software modifications have allowed for better soft tissue balancing
[50-56]. In our experience, when navigational aids were used, $85 \%$ of the implanted knee joints had 'ideal' or 'very good' alignment, compared with $55 \%$ of the implanted knee joints that were fitted with traditional mechanical instrumentation.

\section{Rehabilitation and post-operative thromboprophylaxis}

At our institution, we have adopted a rehabilitation programme that promotes a rapid return to weight-bearing and walking without aids (Table 1). This programme usually results in complete, unaided weight-bearing by 15 days post-operation (range 12-45 days), a return to work by 30 days post-operation (range 20-80 days) and a return to sporting activities from the second month post-operation (range 30-85 days).
Fig. 5 Main surgical steps of TKA assisted by OrthoPilot ${ }^{\mathbb{R}}$ Navigation system (B Braun Medical Ltd, Aesculap): this tool is used to guide the placement of the cutting blocks (a), to verify the accuracy of bone resections and thus mechanical axis alignment (b), and to assist in soft tissue balancing (c)
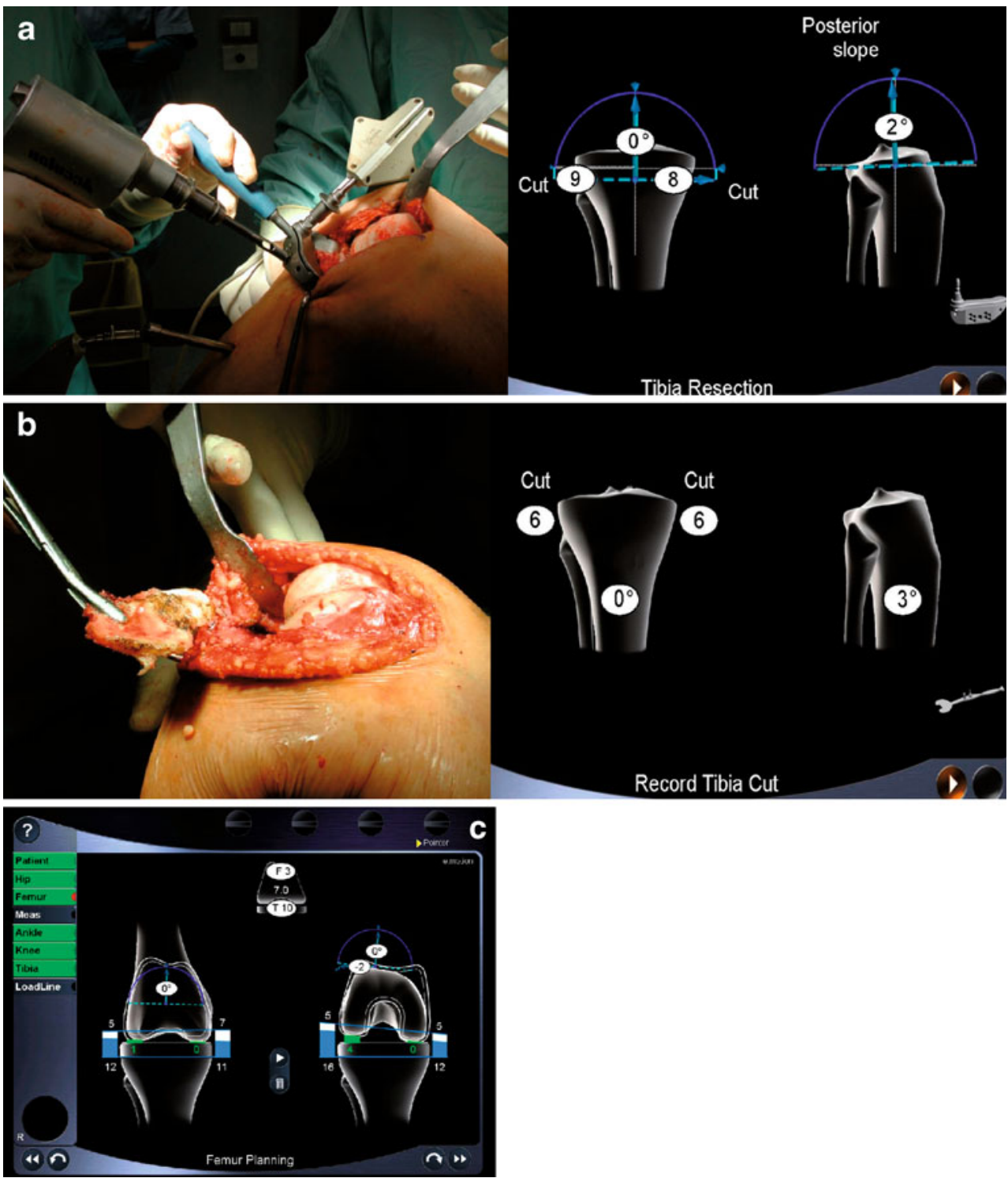
Table 1 An example of a rehabilitation programme used to promote the rapid recovery of knee function following knee replacement surgery

Post-operative period Therapy/activity/exercise Benefits

Phase 1

Days 2-21

Phase 2

Weeks 4-8

Phase 3

Weeks 9+ (third month post-operation) Swimming, cycling and gymnastics

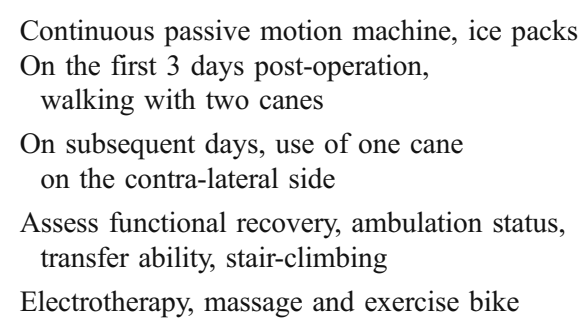

Water-based exercise therapy
This first phase of land-based therapy ensures that the patient regains weight-bearing and mobility as quickly as possible
Particularly beneficial to those patients who have difficulty with land-based, weight-bearing activities

This phase is for strengthening the quadriceps, recovery of proprioception and limb remodelling

Although our patients routinely regain mobility within $24 \mathrm{~h}$ post-operation, adequate post-surgical thromboprophylaxis is still crucial to counter the increased risk of VTE that is associated with surgical procedures [57-59]. A number of agents are recommended by international guidelines, including low molecular weight heparins (LMWHs), the pentasaccharide, fondaparinux and vitamin $\mathrm{K}$ antagonists such as warfarin [60,61]. New oral anticoagulants such as the Factor Xa inhibitor rivaroxaban and direct thrombin inhibitor dabigatran etexilate could address the large proportion of patients who do not receive an adequate duration of thromboprophylaxis [62]. Recently, dabigatran etexilate has been granted a license for primary prevention of VTE following elective total hip replacement or knee replacement surgery [63]. This new oral agent provides a convenient fixed-dose alternative to existing thromboprophylactic drugs. In particular, the replacement of routinely used parenteral LMWH by orally administered dabigatran etexilate facilitates post-hospital discharge thromboprophylaxis in a setting where patients spend less time in hospital because of the use of the new, more effective surgical technologies described in this article.

\section{Conclusions}

The anatomy of the knee, the largest joint in the body, is very complicated. Its role as one of the main weightbearing joints exposes it to the risk of injury and osteoarthritic degeneration. When the pain associated with osteoarthritis becomes unmanageable with non-surgical interventions, then total or partial knee replacement may be the most appropriate treatment option. There are a wide range of surgical techniques and prosthetic components available that allow orthopaedic surgeons the opportunity to replace part or all of the surfaces of the knee joint based on the extent of the disease involvement. MIS techniques provide several advantages over traditional techniques, such as the requirement for less muscle dissection, shorter incisions, less blood loss, less pain, a shorter hospital stay, faster rehabilitation and a more rapid improvement in the range of motion.

Many factors can influence the success of knee replacement surgery, including patient selection, prosthesis design, the extent of the damage to the joint, the accuracy of the surgical technique in terms of soft tissue balancing and limb alignment, and the effectiveness of the post-operative rehabilitation programme [46]. The use of unicompartmental or bi-unicompartmental prostheses in the knee, especially those that require only minimal bone removal, requires careful insertion into the complex biomechanical and kinematic situation of the knee, and evidence of success from long-term studies is somewhat limited. In this case, and especially when bi-unicompartmental prostheses are also used, the prosthesis does not substitute for the joint but integrates with it. In fact, when implanting a UKR, it is wrong to correct the joint biomechanics that caused the pathology; instead, one simply substitutes, according to Romagnoli [35], the part that degenerated due to the disease (resurfacing partial knee arthroplasty). The cruciate-retaining knee bicompartmental arthroplasties, even if not common, seem to offer a high level of functionality and a joint kinematic that presents essential features similar to a normal knee, and a survivor rate comparable to TKA. Bi-unicompartmental arthroplasty has shown an average range of motion of $126^{\circ}$, higher 
than the average standard total knee replacement [35]. UKR plus PF represents a further improvement in technique with good prospects for the future.

By providing intra-operative, real-time information on the location of the joint centres, the position of the prosthetic implants and the alignment of the limb, CAS navigation systems are helping orthopaedic surgeons to implant total replacement knees that have a more anatomically correct mechanical axis. Although there are no long-term outcome data on CAS navigation in TKR, the evidence suggests that it should improve outcomes as a result of the improved implant positioning. High-flex TKA and gender solution represent further elements to meet the needs of young patients in whom other partial resurfacing solutions are not possible when extensive disease involves all three knee compartments. Finally, rehabilitation and thromboprophylaxis in the postoperative period are key components of a well-managed, post-operative care programme, which is essential for the recovery of normal knee function following knee replacement surgery and for minimising the surgery-related increased risk of VTE.

Acknowledgements This work was supported by Boehringer Ingelheim. Writing and editorial assistance was provided by Louise Norbury and Rebecca Gardner PhD of PAREXEL, which was contracted by Boehringer Ingelheim for these services. The author meets criteria for authorship as recommended by the International Committee of Medical Journal Editors (ICMJE), was fully responsible for all content and editorial decisions, and was involved at all stages of manuscript development. The author received no compensation related to the development of the manuscript.

Conflict of interest The author declares no conflict of interest.

Open Access This article is distributed under the terms of the Creative Commons Attribution Noncommercial License which permits any noncommercial use, distribution, and reproduction in any medium, provided the original author(s) and source are credited.

\section{References}

1. Rand JA, Ilstrup DM (1991) Survivorship analysis of total knee arthroplasty. Cumulative rates of survival of 9200 total knee arthroplasties. J Bone Joint Surg Am 73:397-409

2. Ritter MA, Herbst SA, Keating EM, Faris PM, Meding JB (1994) Long-term survival analysis of a posterior cruciate-retaining total condylar total knee arthroplasty. Clin Orthop Relat Res 309:136145

3. Borus T, Thornhill T (2008) Unicompartmental knee arthroplasty. J Am Acad Orthop Surg 16:9-18

4. Jamali AA, Scott RD, Rubash HE, Freiberg AA (2009) Unicompartmental knee arthroplasty: past, present, and future. Am J Orthop (Belle Mead NJ) 38:17-23

5. Yang S, Hadlow S (2003) Unicompartmental knee arthroplasty: is it durable? N Z Med J 116:U627
6. Berger RA, Meneghini RM, Jacobs JJ, Sheinkop MB, Della Valle CJ, Rosenberg AG, Galante JO (2005) Results of unicompartmental knee arthroplasty at a minimum of ten years of follow-up. J Bone Joint Surg Am 87:999-1006

7. Newman J, Pydisetty RV, Ackroyd C (2009) Unicompartmental or total knee replacement: the 15-year results of a prospective randomised controlled trial. J Bone Joint Surg Br 91:52-57

8. Laurencin CT, Zelicof SB, Scott RD, Ewald FC (1991) Unicompartmental versus total knee arthroplasty in the same patient. A comparative study. Clin Orthop Relat Res 273:151-156

9. Dalury DF, Fisher DA, Adams MJ, Gonzales RA (2009) Unicompartmental knee arthroplasty compares favorably to total knee arthroplasty in the same patient. Orthopedics; 32 pii. Available at http://www.orthosupersite.com/view.asp?rID=38057. Accessed 5 November 2010

10. Laskin RS (2004) Minimally invasive total knee replacement using a mini-mid vastus incision technique and results. Surg Technol Int 13:231-238

11. Laskin RS (2005) Minimally invasive total knee arthroplasty: the results justify its use. Clin Orthop Relat Res 440:54-59

12. Leopold SS (2009) Minimally invasive total knee arthroplasty for osteoarthritis. N Engl J Med 360(17):1749-1758

13. Bonutti P (2009) Suspended leg approach and arthroscopicassisted techniques. In: Scuderi GR, Tria AJ (eds) Minimally invasive surgery in orthopaedics. Springer, New York, pp 301-307

14. Schroer WC, Diesfeld PJ, Reedy ME, LeMarr AR (2008) Minisubvastus approach for total knee arthroplasty. J Arthroplasty 23:9-25

15. King J, Stamper DL, Schaad DC, Leopold SS (2007) Minimally invasive total knee arthroplasty compared with traditional total knee arthroplasty. Assessment of the learning curve and the postoperative recuperative period. J Bone Joint Surg Am 89:1497-1503

16. Khanna A, Gougoulias N, Longo UG, Maffulli N (2009) Minimally invasive total knee arthroplasty: a systematic review. Orthop Clin North Am 40:479-489

17. Cheng T, Liu T, Zhang G, Peng X, Zhang X (2010) Does minimally invasive surgery improve short-term recovery in total knee arthroplasty? Clin Orthop Relat Res 468:1635-1648

18. Kozinn SC, Scott R (1989) Unicondylar knee arthroplasty. J Bone Joint Surg Am 71:145-150

19. Swienckowski JJ, Pennington DW (2004) Unicompartmental knee arthroplasty in patients sixty years of age or younger. J Bone Joint Surg Am 86(Suppl 1):131-142

20. Newman JH, Ackroyd CE, Shah NA (2001) Unicompartmental or total knee replacement? J Bone Joint Surg Br 80:862-865

21. Repicci JA (2003) Mini-invasive knee unicompartmental arthroplasty: bone-sparing technique. Surg Technol Int 11:282

22. Banks SA, Frely BJ, Boniforti F, Reischmidt C, Romagnoli S (2005) Comparing in vivo kinematics of unicondylar and biunicondylar knee replacement. Knee Surg Sports Traumatol Arthrosc 13:551-556

23. Fuchs S, Tibesku CO, Frisse D, Genkinger M, Laaß H, Rosenbaum D (2005) Clinical and functional of uni- and bicondylar sledge prostheses. Knee Surg Sports Traumatol Arthrosc 13:197-202

24. Fuchs S, Frisse D, Tibesku CO, Genkinger M, Laaß H, Rosenbaum D (2002) Proprioceptive function, clinical results and quality of life after unicondylar sledge prostheses. Am J Phys Med Rehab 81:478-482

25. Andriacchi TP, Andersson GB, Fermier RW, Stern D, Galante JO (1980) A study of lower-limb mechanics during stair-climbing. J Bone Joint Surg Am 62:749-757

26. Weale AE, Halabi OA, Jones PW, White SH (2001) Perceptions of outcomes after unicompartmental and total knee replacements. Clin Orthop 382:143-153 
27. Patil S, Colwell CW, Ezet KA, D’Lima DD (2005) Can normal knee kinematics be restored with unicompartmental knee replacement? J Bone Joint Surg Am 87:332-338

28. Confalonieri N, Manzotti A, Pullen C (2004) Comparison of a mobile with a fixed tibial bearing unicompartmental knee prosthesis: a prospective randomized trial using a dedicated outcome score. Knee 11:357-362

29. Emerson RH Jr, Hansborough T, Reitman RD, Ros W, Higgins LL (2002) Comparison of a mobile with a fixed-bearing unicompartmental knee implant. Clin Orthop 404:62-70

30. McAlindon TE, Snow S, Cooper C, Dieppe PA (1992) Radiographic patterns of osteoarthritis of the knee joint in the community: the importance of the patellofemoral joint. Ann Rheum Dis 51:844-849

31. Witvoet J (2005) Should patellofemoral prostheses still be used for the treatment of isolated patellofemoral osteoarthritis? Eur Instruc Course Lect 7:174-183

32. Cannon A, Stolley M, Wolf B, Amendola A (2008) Patellofemoral resurfacing arthroplasty: literature review and description of a novel technique. Iowa Orthop J 28:42-48

33. Confalonieri N, Manzotti A, Cerveri P, De Momi E (2009) Biunicompartmental versus total knee arthroplasty: a matched paired study with early clinical results. Arch Orthop Trauma Surg 129:1157-1163

34. Romagnoli S, Camera A, Bertolotti M, Arnaldi E (1998) La protesi Bimonocompartimentale con rispetto ricostruzione del LCA, Il Ginocchio, Anno XIV

35. Romagnoli S, Banks SA, Fregly BJ, Boniforti F, Reinschmidt C (2005) Comparing in vivo kinematics of unicondylar and biunicondylar knee replacement. Knee Surg Sports Traumatol Arthrosc 13:551-556

36. Levitan D (2006) Patellofemoral knee replacement effective when following strict indication criteria. Orthop Today Int 9:8

37. Heyse TJ, Khefacha A, Cartier P (2010) UKA in combination with PFR at average 12-year follow-up. Arch Orthop Trauma Surg 130:1227-1230

38. Tria AJ Jr (2010) Bicompartmental arthroplasty of the knee. Instr Course Lect 59:61-73

39. Rolston L, Bresh J, Engh GA, Alois F, Kreuzer S, Nadaudad M, Puri L, Wood D (2007) Bicompartmental knee arthroplasty: a bone-sparing, ligament sparing, and minimally invasive alternative for active patients. Orthopedics 30(8 Suppl):70-73

40. Engh GA (2007) A bicompartmental solution: what the Deuce? Orthopedics 30:770

41. Callahan CM, Drake BG, Heck DA, Dittus RS (1994) Patient outcomes following tricompartmental total knee replacement. A meta-analysis. JAMA 271:1349-1357

42. Li G, Most E, Sultan PG, Schule S, Zayontz S, Park SE, Rubash HE (2004) Knee kinematics with a high-flexion posterior stabilized total knee prosthesis: an in vitro robotic experimental investigation. J Bone Joint Surg Am 86:1721-1729

43. Ritter MA, Harty LD, Davis KE, Meding JB, Berend ME (2003) Predicting range of motion after total knee arthroplasty. Clustering, log-linear regression, and regression tree analysis. J Bone Joint Surg Am 85:1278-1285

44. Plaster RL, Starkman KB, McGee J (2010) The gender solutions Natural-Knee flex system and future directions. Am J Orthop (Belle Mead NJ) 39(6 Suppl):9-12

45. Munzinger UK, Maffiuletti NA, Guggi T, Bizzini M, Preiss S, Drobny T (2010) Five-year results of the Innex total knee arthroplasty system. Int Orthop 34:1159-1165

46. Siston RA, Giori NJ, Goodman SB, Delp SL (2007) Surgical navigation for total knee arthroplasty: a perspective. J Biomech 40:728-735
47. Sharkey PF, Hozack WJ, Rothman RH, Shastri S, Jacoby SM (2002) Insall Award paper. Why are total knee arthroplasties failing today? Clin Orthop Relat Res 404:7-13

48. Callaghan JJ, O'rourke MR, Saleh KJ (2004) Why knees fail: lessons learned. J Arthroplasty 19(Suppl 1):31-34

49. Stulberg SD, Loan P, Sarin V (2002) Computer-assisted navigation in total knee replacement: results of an initial experience in thirty-five patients. J Bone Joint Surg Am 84 (Suppl 2):90-98

50. Delp SL, Stulberg SD, Davies B, Picard F, Leitner F (1998) Computer assisted knee replacement. Clin Orthop Relat Res $354: 49-56$

51. Zanasi S (2006) Minimally invasive computer-assisted total knee arthroplasty through a subvastus approach. Orthopedics 29(10 Suppl):S142-S144

52. Bäthis H, Perlick L, Tingart M, Lüring C, Zurakowski D, Grifka J (2004) Alignment in total knee arthroplasty. A comparison of computer-assisted surgery with the conventional technique. J Bone Joint Surg Br 86:682-687

53. Chauhan SK, Scott RG, Breidahl W, Beaver RJ (2004) Computerassisted knee arthroplasty versus a conventional jig-based technique. A randomised, prospective trial. J Bone Joint Surg $\mathrm{Br}$ 86:372-377

54. Haaker RG, Stockheim M, Kamp M, Proff G, Breitenfelder J, Ottersbach A (2005) Computer-assisted navigation increases precision of component placement in total knee arthroplasty. Clin Orthop Relat Res 433:152-159

55. Jenny JY, Clemens U, Kohler S, Kiefer H, Konermann W, Miehlke RK (2005) Consistency of implantation of a total knee arthroplasty with a non-image-based navigation system: a case-control study of 235 cases compared with 235 conventionally implanted prostheses. J Arthroplasty 20:832-839

56. Laskin RS, Beksaç B (2006) Computer-assisted navigation in TKA: where we are and where we are going. Clin Orthop Relat Res 452:127-131

57. Hirsh J, Hoak J (1996) Management of deep vein thrombosis and pulmonary embolism. A statement for healthcare professionals. Council on Thrombosis (in consultation with the Council on Cardiovascular Radiology), American Heart Association. Circulation 93:2212-2245

58. Ginsberg JS (1996) Management of venous thromboembolism. N Engl J Med 335:1816-1828

59. Pineo GF, Hull RD (1999) Prophylaxis of venous thromboembolism following orthopedic surgery: mechanical and pharmacological approaches and the need for extended prophylaxis. Thromb Haemost 82:918-924

60. (2007) American Academy of Orthopaedic Surgeons clinical guideline on prevention of symptomatic pulmonary embolism in patients undergoing total hip or knee arthroplasty. Summary of recommendations. Available at http://www.aaos.org/research/ guidelines/PE_summary.pdf. Accessed 22 June 2010

61. Geerts WH, Bergqvist D, Pineo GF, Heit JA, Samama CM, Lassen MR, Colwell CW, American College of Chest Physicians (2008) Prevention of venous thromboembolism: American College of Chest Physicians Evidence-Based Clinical Practice Guidelines (8th Edition). Chest 133(6 Suppl):381S-453S

62. Borris LC (2010) Rivaroxaban and dabigatran etexilate: two new oral anticoagulants for extended postoperative prevention of venous thromboembolism after elective total hip arthroplasty. Arch Orthop Trauma Surg 130:583-589

63. Pradaxa ${ }^{\circledR}$ Summary of Product Characteristics. 2009; Boehringer Ingelheim International $\mathrm{GmbH}$. Available at http://emc.medicines. org.uk/medicine/20760. Accessed 5 November 2010 